\title{
AVALIAÇÃO DO NÍVEL DE COOPERAÇÃO DE PACIENTES EM TERAPIA PERIODONTAL DE SUPORTE
}

Manoel Cirilo Silva Neto

Cirurgião-Dentista.

Samantha Ariadne Alves Freitas

Doutoranda em Odontologia; Programa de Pós-Graduação em Odontologia; Universidade Federal do Maranhão (UFMA), Brasil.

Camila Lima Duailibe

Discente de graduação em Odontologia da Universidade Federal do Maranhão (UFMA), Brasil.

\section{Liana Linhares Lima Serra}

Doutora em Odontologia; Docente Adjunta na Universidade Federal do Maranhão (UFMA), Brasil.

\section{Antonio Luiz Amaral Pereira}

Doutor em Patologia Bucal; Docente Associado na Universidade Federal do Maranhão (UFMA), Brasil.

\section{Adriana de Fátima Vasconcelos Pereira}

Doutora em Odontologia; Docente Adjunta do Departamento de Odontologia II, Universidade Federal do Maranhão (UFMA), Brasil.

E-mail: adriana.ufma@hotmail.com
RESUMO: A motivação e a educação em saúde bucal são consideradas ferramentas de base para a prevenção das doenças periodontais. $\mathrm{O}$ objetivo deste estudo foi avaliar o nível de cooperação dos pacientes da Clínica de Periodontia do Curso de Odontologia da Universidade Federal do Maranhão durante a Terapia Periodontal de Suporte (TPS). Avaliou-se clinicamente a profundidade de sondagem (PS), recessão gengival (RG), nível de inserção clínica (NIC), mobilidade dentária, envolvimento de furca, índice de placa (IP) e índice de sangramento gengival (ISG) de 16 pacientes adultos em três fases: baseline, T1 e T2. Os indivíduos foram diagnosticados quanto à condição periodontal e responderam ao questionário sobre o impacto na rotina e qualidade de vida nos últimos seis meses pelo índice OHIP-14 (Oral Health Impact Profile). Os dados foram submetidos à análise estatística. Os pacientes foram classificados conforme o grau de cooperação em: colaboradores (75\%), colaboradores irregulares (12,5\%) e não colaboradores $(12,5 \%)$. A PS e o NIC dos pacientes colaboradores durante as três fases não sofreram diferenças significativas. O ISG apresentou redução quando comparado ao baseline. A média do IP dos pacientes colaboradores evidenciou uma pequena redução, ao contrário dos colaboradores irregulares. Não houve escores de impacto forte na avaliação do OHIP. 14, demonstrando uma boa percepção da qualidade da saúde bucal pelos indivíduos. Os pacientes colaboradores tiveram um impacto fraco em relação à doença periodontal nas duas fases do estudo (T1 e T2). Os principais diagnósticos foram a gengivite associada ao biofilme $(38,46 \%)$ e periodontite crônica avançada localizada (30,76\%). Pode-se concluir que os pacientes, em sua maioria, mostraram-se colaboradores na TPS, mantendo sua condição periodontal estável ou progredindo para saúde periodontal.

PALAVRAS-CHAVE: Cooperação do Paciente; Doenças Periodontais; Motivação; Qualidade de Vida.

\section{ASSESSMENT OF PATIENTS' COOPERATION LEVEL IN BASIC PERIODONTAL THERAPY}

ABSTRACT: Motivation and education in mouth health are basic tools to prevent periodontal diseases. Current study assesses patients' cooperation level at the Periodontal Clinic of the Dentistry Course of the Universidade Federal do Maranhão during Basic Periodontal Therapy (BPT). Sounding depth (PS), gum recession (RG), level of clinical insertion, dental mobility, furca involvement, plaque index (IP) and gum blooding index (ISG) of six adult were assessed clinically at three phases: baseline, T1 and T2. The patients were diagnosed with regard to their periodontal condition and answered a questionnaire on the impact on routine and life quality during the last six months, using Oral Health Impact Profile (OHIP-14). Data underwent statistical 
analysis and patients were classified according to their cooperation level, namely, collaborators (75\%), irregular collaborators (12.5\%) and non-collaborators (12.5\%). No significant differences existed in collaborators' PS and NIC during the three phases. ISG decreased when compared to baseline. IP mean of collaborating patients showed a slight decrease when compared to that of irregular collaborators. Since no significant score impact existed in OHIP-14 assessment, there was a good perception by patients with regard to mouth health quality. Collaborating patients had a weak impact with regard to periodontal disease in the two phases (T1 and T2). Main diagnoses were gingivitis plus biofilm gum $(38.46 \%)$ and local advanced chronic periodontitis (30.76\%). Results show that most patients collaborated with BPT, with a stable or advanced periodontal condition in periodontal health.

KEY WORDS: Periodontal Diseases; Motivation; Patient's Cooperation; Life Quality.

\section{INTRODUÇÃO}

As doenças periodontais são um grupo de doenças inflamatórias de origem infecciosa, que afetam os tecidos de proteção e sustentação dos dentes (ARMITAGE, 1999), resultantes da interação entre biofilme dentário e resposta imune do hospedeiro (MARSH, 2004; SOCRANSKY; HAFFAJEE, 2005).

A terapia periodontal tem o propósito de preservar os dentes por meio da resolução do processo inflamatório e evitar a progressão da doença (FAMILI; SHORT, 2010; SAMINSKY et al., 2015). Dentro desse contexto, a Terapia Periodontal de Suporte (TPS) visa à conservação dos resultados obtidos na fase associada à causa (DANTAS et al., 2011; PEREIRA et al., 2011). Essa terapia denota a necessidade básica dos procedimentos terapêuticos para que os pacientes, por meio de esforços próprios, possam controlar a infecção periodontal (LINDHE; LANG; KARRING, 2010).

Os objetivos da TPS são: minimizar a recorrência e a progressão da doença periodontal em pacientes que tenham sido tratados previamente de gengivite e periodontite; reduzir a incidência de perda dentária e reabilitação com próteses; aumentar a probabilidade de localizar e tratar, periodicamente, outras doenças e condições encontradas na cavidade bucal (AMERICAN ACADEMY OF PERIODONTOLOGY, 2003; GRANDE et al., 2007) e manter funcionalmente e esteticamente a dentição (RENVERT; PERSSON, 2004).

A motivação e a educação em saúde bucal são consideradas ferramentas de base para a prevenção das doenças periodontais, que deve incluir informações sobre a etiopatogênese, assim como princípios básicos e métodos de controle (BUISCHI, 2000). O determinante clínico de maior importância na terapia periodontal não seria a técnica (cirúrgica ou não cirúrgica), utilizada na eliminação da infecção subgengival, mas sim a qualidade do programa de motivação, de manutenção e de controle (PADOVANI; SABA-CHUJFI, 1991).

Em relação à duração das sessões da TPS, devese adaptar conforme a atividade da doença em cada paciente. Contudo, o intervalo de consulta de retorno para pacientes no primeiro ano de tratamento não deveria ser maior que três meses (CARRANZA JR. et al., 2011). Outros intervalos de tempo podem ser estabelecidos para o retorno periódico dos pacientes em terapia periodontal de suporte, tais como: 1 mês ou 3 a 4 meses (AMERICAN ACADEMY PERIODONTOLOGY, 2003; SUBRAMANIUM et al., 2014).

Alguns indicadores sócio-dentários de qualidade de vida são utilizados para pesquisas de saúde bucal, os quais podem ser aplicados em estudos com pacientes inseridos em um programa de manutenção. Um dos índices mais utilizados é o Oral Health Impact Profile - OHIP-14 (MIOTTO; BARCELLOS; VELTEN, 2012), cuja versão simplificada já está validada no Brasil (OLIVEIRA; NADANOVSKY, 2005).

Diante do exposto, este estudo teve o objetivo de avaliar o nível de cooperação e autopercepção da condição periodontal dos pacientes da Clínica de Periodontia do Curso de Odontologia da Universidade Federal do Maranhão (UFMA) durante a Terapia Periodontal de Suporte.

\section{METODOLOGIA}

Foram atendidos 99 pacientes, no $1^{0}$ semestre de 2014, na Clínica de Periodontia da Faculdade de 
Odontologia da UFMA, de ambos os gêneros e na faixa etária de 20 a 72 anos. No entanto, foram excluídos os pacientes menores de 18 anos de idade $(n=2)$, aqueles que não concluíram o tratamento $(n=42)$, não aceitaram participar $(n=3)$, bem como os casos nos quais não foi possível entrar em contato/números inexistentes $(\mathrm{n}=32)$ e aqueles que foram marcados, mas não compareceram $(\mathrm{n}=4)$, totalizando uma seleção de 16 pacientes adultos para este estudo.

Os pacientes receberam informações verbais e por escrito sobre a pesquisa, sendo que aqueles que concordaram em participar assinaram o Termo de Consentimento Livre e Esclarecido. Este estudo foi aprovado pelo Comitê de Ética e Pesquisa do Hospital Universitário da Universidade Federal do Maranhão sob o Parecer n ${ }^{\circ} 275.507$.

Os pacientes foram convidados a retornar à faculdade para uma reavaliação quanto às condições periodontais, tais como: índice de placa, índice de sangramento à sondagem, profundidade de sondagem, recessão gengival, nível de inserção clínica, mobilidade dentária, envolvimento de furca e perda dentária. Os fumantes foram classificados de acordo com a quantidade de cigarros fumados por dia (leve: $<10$ cigarros/dia ou Pesado: $>10$ cigarros/dia) e ao tempo do hábito (curta duração: $<10$ anos de hábito ou longa duração: $>10$ anos de hábito) (HABER; KENT, 1992).

Para a classificação dos diabéticos, tomou-se por base a diabetes em tipos I e II e, na oportunidade, foi solicitado o último exame laboratorial (glicemia em jejum ou hemoglobina glicada) para verificar se os mesmos estavam com a doença dentro dos padróes de normalidade ou controle. Esses dados foram anotados em ficha clínica elaborada para a pesquisa, sendo ainda aplicado um questionário socioeconômico, além de os pacientes terem também respondido às perguntas relacionadas aos seus hábitos de higiene bucal, saúde geral e hábitos de vida.

Quanto ao nível de cooperação, os 16 pacientes foram classificados em três grupos (DEMIREL; EFEODLU, 1995): colaboradores (pacientes que retornam regulamente a todas as consultas de controle e manutenção), colaboradores irregulares (pacientes que retornam irregularmente às consultas, ou seja, que faltam em duas ou mais consultas) ou não colaboradores (pacientes que retornaram à primeira consulta, mas que abandonaram o programa).

O estudo foi realizado em três etapas: primeira fase ou baseline (T0), segunda fase (T1) e terceira fase (T2). Os pacientes que concluíram o tratamento no baseline retornaram em mais dois momentos para TPS (T1 e T2).

\subsection{PRIMEIRA FASE OU BASELINE (T0)}

Constituiu na seleção de fichas clínicas dos pacientes que haviam concluído tratamento periodontal na clínica de Periodontia da UFMA para coleta de dados referentes aos parâmetros periodontais de profundidade de sondagem, recessão gengival, nível de inserção clínica, mobilidade dentária e envolvimento de furca.

Em seguida, os pacientes foram diagnosticados e procedeu-se à classificação desses achados com base nas seguintes condições em: saúde periodontal, gengivite associada ao biofilme dentário, periodontite crônica leve/moderada/avançada (quanto à gravidade) e periodontite crônica localizada/generalizada (quanto à extensão) (ARMITAGE, 1999). Ademais, um protocolo de tratamento periodontal não cirúrgico foi instituído contemplando: orientação de higiene bucal, raspagem e alisamento radicular, polimento e aplicação tópica de flúor. Acrescido ainda pelo informe aos pacientes acerca da importância de sua colaboração com a manutenção da higiene bucal ao longo de todo o tratamento.

\subsection{SEGUNDA FASE OU T1}

Foi realizada a primeira reavaliação (3 meses após a terapia básica) com obtenção de dados sobre os parâmetros clínicos e diagnósticos periodontais. Os pacientes que apresentaram na reavaliação sítios com sangramento à sondagem, presença significativa de biofilme dental e cálculo, perda de inserção clínica, envolvimento de furca e mobilidade dentária foram submetidos a novo tratamento periodontal não cirúrgico.

Nesta fase, foi reforçado o processo de motivação ao autocuidado com os pacientes, incluindo explicação sobre a importância da TPS, sobre as doenças periodontais 
e nova orientação de higiene bucal. Foram entregues aos pacientes escovas dentárias.

O índice subjetivo OHIP-14 (Oral Health Impact Profile) foi aplicado no formato de entrevista. Este índice abordou as dimensões sociais, psicológicas e funcionais afetadas por doenças bucais (OLIVEIRA; NADANOVSKY, 2005) por meio de 14 perguntas divididas em sete escalas (SILVA; FERNANDES, 2001).

Devido à necessidade de investigar a influência da doença periodontal na qualidade de vida desses pacientes, acrescentou-se a palavra "gengiva" em 13 perguntas do índice, exceto na pergunta $n^{\circ} 3$ (DRUMOND-SANTANA et al., 2007). Para calcular o valor individual do OHIP-14 foi utilizado o método ponderado padronizado. Este método leva em conta o peso de cada questão e multiplica pela codificação da pergunta, conforme a escala do tipo Likert (Nunca $=0$; Raramente $=1$; Às vezes $=2$; Frequentemente $=3$; Sempre $=4$; Não sabe $=$ exclusão de todo o formulário).

$\mathrm{O}$ peso de cada item foi gerado pelo método de comparação pareada de Thurstone derivada do estudo de Slade (1997). O somatório de todas as perguntas para cada indivíduo variou entre 0 e 28 . O impacto para cada um dos pacientes foi classificado em fraco ( 0 a 9), médio (10 a 18) e forte (19 a 28) (BASTOS, 2009). Como as respostas poderiam dar fracionadas, foram considerados os valores menores que 9,33 como pertencentes ao grupo fraco; valores entre 9,33 e menores que 18,66 pertencentes ao grupo médio; e entre 18,66 e 28 como grupo forte.

\subsection{TERCEIRA FASE OU T2}

Constituiu o momento de retorno dos pacientes para uma nova reavaliação após 60 dias do T1. Decorrido este período de tempo, foram avaliados os resultados da motivação ao autocuidado e do tratamento não cirúrgico. Novo exame clínico periodontal foi realizado e aqueles pacientes que apresentaram, na reavaliação, quantidade significativa de biofilme dentário e cálculo supragengival e subgengival, sítios reinfectados com sangramento à sondagem, profundidade de sondagem, grande perda de inserção clínica, envolvimento de furca e mobilidade dentária foram submetidos a novo tratamento periodontal não cirúrgico baseado no perfil de cada paciente. Foram entregues aos pacientes folders explicativos (Colgate) e escovas dentárias.

\section{RESULTADOS}

A Tabela 1 representa a associação das variáveis demográficas, sociais e biológicas dos pacientes na primeira fase ou baseline. Observou-se que a amostra foi composta por 12 indivíduos do gênero feminino (75\%) e 4 do gênero masculino (25\%), com faixa etária entre 20 e 72 anos. Em relação ao hábito de fumar, $93,75 \%$ dos pacientes eram não fumantes e $6,25 \%$ eram fumantes. $O$ diagnóstico periodontal mais encontrado foi: $56,25 \%$ dos pacientes apresentando gengivite associada ao biofilme dentário e $18,75 \%$ com periodontite crônica avançada localizada.

Tabela 1. Caracterização da Amostra no Baseline (São Luís, MA, 2015)

(continua)

\begin{tabular}{lll}
\hline Variáveis & $\mathbf{n}$ & $\%$ \\
\hline Idade & & \\
\hline 20 a 39 & 6 & $37,5 \%$ \\
40 a 72 & 10 & $62,5 \%$ \\
\hline Gênero & & \\
\hline Feminino & 12 & $75 \%$ \\
Masculino & 7 & $25 \%$ \\
\hline Estado Civil & & \\
\hline Solteiro & 7 & $43,75 \%$ \\
Casado & 8 & $50 \%$ \\
Divorciado & 1 & $6,25 \%$ \\
\hline Hábito de Fumar & & \\
\hline Não Fumante & 15 & $93,75 \%$ \\
Fumante & 1 & $6,25 \%$ \\
\hline Doenças Sistêmicas & & \\
\hline Normorreativo & 10 & $62,5 \%$ \\
Hipertensão & 3 & $18,75 \%$ \\
Diabetes & 1 & $6,25 \%$ \\
Tetraplegia e Epilepsia & 1 & $6,25 \%$ \\
Artrose & 1 & $6,25 \%$ \\
\hline Diagnóstico Periodontal & & \\
\hline Saúde Periodontal & & $0,0 \%$ \\
& & \\
\hline & & \\
\hline
\end{tabular}




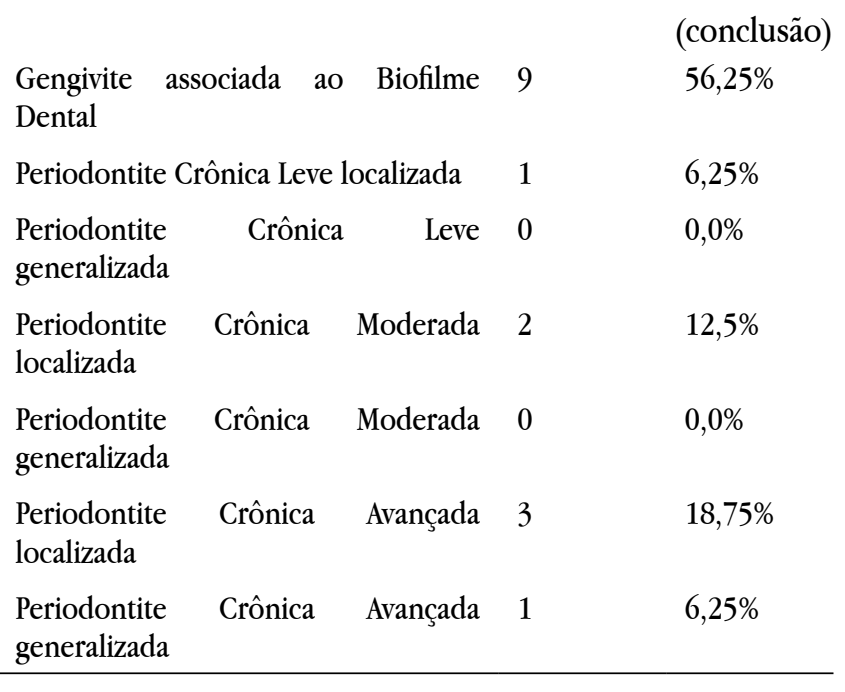

A Tabela 2 mostra a relação entre o OHIP-14 e a influência da doença periodontal na rotina e qualidade de vida dos pacientes colaboradores, colaboradores irregulares e não colaboradores na segunda fase ou T1. Dos 16 pacientes, nenhum obteve um valor de OHIP14 forte, ficando a amostra composta por impacto fraco $(68,75 \%)$ e médio $(31,25 \%)$ na qualidade de vida. A amostra variou na pontuação individual do OHIP-14 de 0 a 13,4. Observou-se que em todos os níveis de percepção houve maior porcentagem de indivíduos com impacto fraco, sendo a maioria do grupo dos colaboradores (75\%).

Tabela 2. Relação entre o OHIP-14 e o Grau de Cooperação dos Pacientes em T1 (São Luís, MA, 2015)

\begin{tabular}{|c|c|c|c|c|c|c|c|c|}
\hline & \multicolumn{8}{|c|}{ OHIP-14 } \\
\hline & \multicolumn{2}{|c|}{ Fraco (0 a 9) } & \multicolumn{2}{|c|}{ Médio (10 a 18) } & \multicolumn{2}{|c|}{ Forte (19 a 28$)$} & \multicolumn{2}{|c|}{ Total } \\
\hline & $\mathrm{n}$ & $\%$ & $\mathrm{n}$ & $\%$ & $\mathrm{n}$ & $\%$ & $\mathrm{n}$ & $\%$ \\
\hline Colaboradores & 9 & $75 \%$ & 3 & $25 \%$ & 0 & $0 \%$ & 12 & $100 \%$ \\
\hline Colaboradores Irregulares & 2 & $100 \%$ & 0 & $0 \%$ & 0 & $0 \%$ & 2 & $100 \%$ \\
\hline Não Colaboradores & 0 & $0 \%$ & 2 & $100 \%$ & 0 & $0 \%$ & 2 & $100 \%$ \\
\hline
\end{tabular}

A relação entre o OHIP-14 e a influência da doença periodontal na rotina e qualidade de vida dos pacientes na terceira fase revelou que o impacto fraco do OHIP-14 subiu de 9 (75\%) para $10(90,9 \%)$ pacientes, apresentando uma diferença percentual de $15,9 \%$, no grupo colaborador na terceira fase ou T2. Já o impacto médio, no mesmo grupo, diminuiu de 3 pacientes $(25 \%$ - T1) para 1 (9,09\% - T2), apresentando uma diferença percentual de 15,91\%. Não houve diferença do OHIP-14 na rotina e qualidade de vida do grupo dos colaboradores irregulares.

Foram avaliados os dados de profundidade de sondagem (PS), nível de inserção clínica (NIC) e recessão gengival $(\mathrm{RG})$ dos pacientes colaboradores no baseline. Considerou-se todos pacientes como colaboradores nessa primeira consulta. A PS-faces livres apresentou média de 7,78 e OS-faces proximais média de 7,62. O NIC-faces livres apresentou média de 7,78 e NIC-faces proximais média de 7,74. A RG-faces livres apresentou média de 1,62 e RG-faces proximais média de 0,54.

$\mathrm{Na}$ Tabela 3, observam-se os valores de índice de placa (IP) e índice de sangramento gengival (ISG) dos pacientes colaboradores no baseline. Em relação ao IP, as faces proximais apresentaram as maiores médias, sendo a face mesial com 22,14 e a distal com 20,47. O percentual total de faces coradas foi de $72,30 \%$. No ISG, os sítios mésio-palatino (6,63\%), disto-palatino (6,35\%) e mésiovestibular (6,32\%) apresentaram maior sangramento à sondagem. $O$ percentual total de sítios com sangramento gengival foi de 33,61\%. A mobilidade dentária grau 2 apresentou a maior média $(0,37)$, mas sem grande significância em relação à amostra total de indivíduos. Apenas a lesão de furca grau I apresentou média de 0,31.

Tabela 3. IP, ISG, Mobilidade Dentária e Lesão de Furca dos Pacientes Colaboradores na Primeira Fase ou Baseline (São Luís, MA, 2015)

(continua)

\begin{tabular}{lcc}
\hline Variável & V & Baseline \\
\hline \multirow{4}{*}{ IP } & V & 12,61 \\
& P & 17,10 \\
& M & 22,14 \\
& D & 20,47 \\
& IP(\%) & $72,30 \%$ \\
\hline
\end{tabular}




\begin{tabular}{lcc} 
& & (conclusão) \\
\hline \multirow{4}{*}{ ISG } & DV & 6,04 \\
& V & 5,00 \\
& MV & 6,32 \\
& DP & 6,35 \\
& P & 5,91 \\
MP & 6,63 \\
Modalidade Dentária & ISG(\%) & $33,61 \%$ \\
\hline \multirow{3}{*}{ Lesão de Furca } & 1 & 0,25 \\
& 2 & 0,37 \\
& 3 & 0,18 \\
\hline
\end{tabular}

Valores da Tabela $=$ Média aritmética $/ \mathrm{V}=$ vestibular, $\mathrm{P}=$ palatina, $\mathrm{M}=$ mesial, $\mathrm{D}=$ distal, $\mathrm{DV}=$ disto-vestibular, $\mathrm{MV}=$ mésio-vestibular, $\mathrm{DP}=$ disto-palatino, $\mathrm{MP}=$ mésio-palatino.
Foram observados os parâmetros periodontais dos pacientes colaboradores, colaboradores irregulares e não colaboradores na primeira fase ou T1. Não houve diferença para PS e NIC dos colaboradores e colaboradores irregulares em nenhuma das faces. Já para o grupo dos não colaboradores, houve diferença em relação aos demais grupos, tanto nas faces livres como nas proximais. ARG apresentou médias superiores para os colaboradores e colaboradores irregulares e menores médias para o grupo dos não colaboradores.

A Tabela 4 demonstra os parâmetros periodontais (IP, ISG, mobilidade dentária e lesão de furca) dos pacientes na segunda fase ou T1. Os pacientes não colaboradores apresentaram média de IP (79\%) maior do que a dos pacientes colaboradores e colaboradores irregulares. Em relação ao ISG, não houve diferença percentual para nenhum dos grupos. Apenas os pacientes colaboradores apresentaram algum grau de mobilidade dentária e lesão de furca.

Tabela 4. IP, ISG, Mobilidade Dentária e Lesão de Furca dos Pacientes em T1 (São Luís, MA, 2015)

\begin{tabular}{lcccc}
\hline Variável & & Colaboradores & Colaboradores Irregulares & Não Colaboradores \\
\hline & V & 13,35 & 6,09 & 18,15 \\
IP & P & 12,57 & 13,45 & 20,37 \\
& M & 19,49 & 15,5 & 21,5 \\
& D & 19,08 & 11,0 & 19,0 \\
& IP(\%) & $64,49 \%$ & $46,00 \%$ & $79,00 \%$ \\
\hline \multirow{4}{*}{ ISG } & DV & 2,10 & 1,59 & 0,5 \\
& V & 1,45 & 2,29 & 0,5 \\
& MV & 3,34 & 3,77 & 2,09 \\
& DP & 4,00 & 3,5 & 4,8 \\
& P & 3,05 & 4,0 & 5,0 \\
Modalidade Dentária & MP & 5,00 & 4,0 & 5,73 \\
& ISG(\%) & $18,94 \%$ & $19,15 \%$ & $18,62 \%$ \\
\hline \multirow{4}{*}{ Lesão de Furca } & 1 & 0,16 & 0,0 & 0,0 \\
& 2 & 0,41 & 0,0 & 0,0 \\
& I & 0,08 & 0,0 & 0,0 \\
\hline
\end{tabular}

Valores da Tabela $=$ Média aritmética $/ \mathrm{V}=$ vestibular, $\mathrm{P}=$ palatina, $\mathrm{M}=$ mesial, $\mathrm{D}=$ distal, $\mathrm{DV}=$ disto-vestibular, $\mathrm{MV}=$ mésio-vestibular, $\mathrm{DP}=$ disto-palatino, $\mathrm{MP}=$ mésio-palatino. 
Quanto aos parâmetros periodontais (PS, NIC e RG) dos pacientes colaboradores e colaboradores irregulares na terceira fase ou T2, observou-se que tanto os pacientes colaboradores como colaboradores irregulares apresentaram PS nas faces livres e proximais com mesma média, sendo a média de 7,3 para os colaboradores e de 7,5 para os colaboradores irregulares. Em relação ao NIC, ambos os grupos apresentaram valores próximos referentes à média para faces proximais e livres, sendo de 7,29 para os colaboradores e de 7,5 para os pacientes colaboradores irregulares. A RG nas faces livres foi de 1,19 para colaboradores e 1,33 para colaboradores irregulares. A RG nas faces proximais foi de 1,1 para os colaboradores e 0,66 para os colaboradores irregulares.

$\mathrm{Na}$ Tabela 5 podem ser observados os dados sobre os parâmetros periodontais (IP, ISG, mobilidade dentária e lesão de furca) dos pacientes colaboradores e colaboradores irregulares na terceira fase ou T2. Os pacientes colaboradores apresentaram IP (61\%) maior que a do grupo dos colaboradores irregulares (20,26\%). Para o ISG, não houve diferença para os dois grupos. Apenas o grupo dos colaboradores apresentou médias para algum grau de mobilidade dentária e lesão de furca.

Tabela 5. IP, ISG, Mobilidade Dentária e Lesão de Furca dos Pacientes Colaboradores e Colaboradores Irregulares na Terceira Fase ou T2 (São Luís, MA, 2015)

\begin{tabular}{|c|c|c|c|}
\hline Variável & & Colaboradores & Colaboradores Irregulares \\
\hline \multirow{5}{*}{ IP } & $\mathbf{v}$ & 6,35 & 0,0 \\
\hline & $\mathbf{P}$ & 11,87 & 6,26 \\
\hline & M & 22,0 & 8,0 \\
\hline & D & 20,72 & 6,0 \\
\hline & IP(\%) & $61 \%$ & $20,26 \%$ \\
\hline \multirow{7}{*}{ ISG } & DV & 3,0 & 2,19 \\
\hline & $\mathbf{v}$ & 2,0 & 1,34 \\
\hline & MV & 3,93 & 1,98 \\
\hline & DP & 3,35 & 4,8 \\
\hline & $\mathbf{P}$ & 2,60 & 3,5 \\
\hline & MP & 3,96 & 4,86 \\
\hline & ISG(\%) & $18,84 \%$ & $18,67 \%$ \\
\hline \multirow{3}{*}{ Modalidade Dentária } & 1 & 0,63 & 0,0 \\
\hline & 2 & 0,27 & 0,0 \\
\hline & 3 & 0,0 & 0,0 \\
\hline \multirow{3}{*}{ Lesão de Furca } & I & 0,09 & 0,0 \\
\hline & II & 0,0 & 0,0 \\
\hline & III & 0,0 & 0,0 \\
\hline
\end{tabular}

Valores da Tabela $=$ Média aritmética $/ \mathrm{V}=$ vestibular, $\mathrm{P}=$ palatina, $\mathrm{M}=$ mesial, $\mathrm{D}=$ distal, $\mathrm{DV}=$ disto-vestibular, $\mathrm{MV}=$ mésio-vestibular, $\mathrm{DP}=$ disto-palatino, $\mathrm{MP}=$ mésio-palatino.

$\mathrm{Na}$ comparação entre os parâmetros clínica (faces livres e proximais), além de uma redução periodontais dos pacientes colaboradores no baseline, T1 e T2, constatou-se que durante as duas fases de reavaliação periodontal (T1 e T2) houve uma diminuição da recessão gengival (faces livres) em comparação ao baseline.

Quando compara-se os parâmetros periodontais da profundidade de sondagem e um ganho de inserção (IP, ISG, mobilidade dentária e lesão de furca) dos 
pacientes colaboradores no baseline, T1 e T2, observouse que ocorreu uma diminuição do IP nas duas fases do estudo em relação ao baseline. O ISG manteve-se quase igual nas duas fases, mas com uma redução expressiva em comparação ao baseline. A mobilidade dentária grau 2 diminuiu no T2 em relação ao T1 e ao baseline. Na lesão de furca grau I houve redução em comparação ao baseline.

O diagnóstico periodontal dos pacientes colaboradores nas 3 fases revelou que, em sua maioria, os pacientes apresentaram gengivite associada ao biofilme dentário. Deve ser ressaltado que no baseline todos os pacientes demonstraram algum tipo de doença periodontal, contudo nas fases T1 e T2 foi observado também diagnóstico de saúde periodontal.

Após a segunda fase ou T1, não foi relatado pelos pacientes o hábito de fumar. Dos 16 pacientes, apenas um era diabético tipo II controlado.

Em relação ao nível de cooperação, $75 \%$ dos pacientes foram classificados como colaboradores, $12,5 \%$ como colaboradores irregulares e $12,5 \%$ como não colaboradores.

\section{DISCUSSÃO}

A Terapia Periodontal de Suporte é uma extensão do tratamento periodontal, que compreende procedimentos realizados em intervalos regulares de modo a permitir a inclusão durante esta fase dos pacientes previamente tratados de doença periodontal e, em longo prazo, possibilitando a prevenção da recorrência, bem como a progressão da doença (AMERICAN ACADEMY PERIODONTOLOGY, 2003; GUGLIELMETTI et al., 2010), o que justificou a execução desta pesquisa.

Participaram da composição da amostra 16 pacientes, sendo a maioria do gênero feminino (75\%), corroborando com o fato de mulheres serem mais assíduas às consultas e preocupadas com a saúde bucal (COSTA et al., 2014), sendo que tais resultados estão de acordo com estudos os quais também observaram maior prevalência de mulheres durante a realização da TPS (SOUSA; NÓBREGA; ARAKI, 2014; BELLO et al., 2011; SILVA et al., 2010). Outro estudo realizado com
121 pacientes submetidos à TPS demonstrou que 51\% deles pertenciam ao gênero feminino (WEHMEYER et al., 2014). Em relação à faixa etária, os pacientes com mais de 30 anos apresentaram maior incidência de doença periodontal, sem distinção de gênero, nesta pesquisa.

Durante o baseline, os pacientes colaboradores apresentaram média de IP de $72,30 \%$. Na segunda fase (T1), essa média diminuiu para $64,49 \%$ e, na terceira fase (T2), para 61\%. Para os colaboradores irregulares, no T1 o IP era de $46 \%$ e no T2 foi reduzido para 20,26\%. Deve ser considerado que o padrão clínico de IP aceitável é de 20$25 \%$ para a maioria dos pacientes; além do que se torna importante também compreender que a quantidade de biofilme dentro da boca apresenta relação com a resposta do hospedeiro, ou seja, com os parâmetros inflamatórios (LINDHE; LANG; KARRING, 2010; O'LEARY; DRAKE; NAYLOR, 1972). Nesta pesquisa, este padrão condiz com a média dos pacientes colaboradores irregulares, mas não em relação ao grupo colaborador que sofreu uma pequena redução. Talvez pelo fato da motivação variar em cada paciente e pela forma como eles realizam sua higiene bucal no dia a dia.

Neste estudo, observou-se uma redução significativa do ISG dos pacientes colaboradores durante as duas fases de reavaliação periodontal (T1 - 18,94\% e T2 - 18,84\%) quando comparadas ao baseline $(33,61 \%)$, o que revela que pacientes assistidos por meio da TPS demonstram melhoras das condições clínicas e periodontais por meio do controle do biofilme dentário e, por conseguinte, a diminuição da recorrência de doenças periodontais (JONSSON et al., 2012). Estudos evidenciaram que o ISG de 20-30\% representa um valor significativo para progressão da enfermidade e que pacientes com média de porcentagem de ISG maiores do que $20 \%$ apresentavam alto risco de reinfecção (CLAFFEY et al., 1990; BARDERSTEN; NILVÉUS; EGELBERG, 1990; JOSS; ADLER; LANG, 1994; CALVASINA; NUTO, 2009).

Destaca-se que a PS e o NIC dos pacientes colaboradores durante as três fases da pesquisa não sofreram diferenças significativas, mantendo-se estáveis. Segundo Westfelt et al. (1983), os níveis de inserção clínica tornam-se estáveis seis meses após o tratamento periodontal ativo, portanto tem sido sugerido que os primeiros seis meses após o fim da terapia sejam 
considerados uma fase de cicatrização, durante a qual é recomendada a realização de frequentes profilaxias dentárias.

$\mathrm{Na}$ avaliação dos riscos dentários, tem-se a mobilidade dentária que pode ser induzida por dois fatores: lesões traumáticas progressivas e altura óssea; esta avaliação pode ser utilizada durante o prognóstico e função para os dentes, indicando possíveis necessidades de intervenções específicas durante as visitas da Terapia Periodontal de Suporte (LINDHE; LANG; KARRING, 2010).

Os pacientes colaboradores foram diagnosticados no baseline com gengivite associada ao biofilme dentário $(56,25 \%)$ e algum tipo de periodontite (43,75\%). Na segunda fase (T1), um paciente foi diagnosticado com saúde periodontal, 8 com gengivite associada ao biofilme dentário e 7 com algum tipo de periodontite crônica. No T2, houve um paciente com saúde periodontal, $5 \mathrm{com}$ gengivite associada ao biofilme dental e 7 com algum tipo de periodontite crônica. Pacientes com gengivite quando tratados e submetidos à TPS apresentam melhor controle de biofilme e menor perda de inserção quando comparados com pacientes sem acompanhamento periodontal (LORENTZ et al., 2009; LAURELL et al., 1991; VALDERHAUG, 1980). Em pacientes com periodontite crônica, a TPS passa a ter importância fundamental, visto que há pequena parcela em que advém a recorrência da infecção periodontal, principalmente nos casos de periodontite avançada. Este estudo mostrou que essa parcela corresponde a $25 \%$ dos pacientes, ocorrendo em intervalos de tempo imprevisíveis (LINDHE; NYMAN, 1984).

A adesão dos pacientes à TPS foi de $75 \%$. Isso pode ser explicado pelo fato de que os pacientes atendidos na Clínica de Periodontia da UFMA, durante todo o tratamento não cirúrgico, passam por sessões de orientação de higiene bucal, raspagem e alisamento radicular e são ainda motivados a manterem sua saúde bucal ao longo de todo o tratamento. Além disso, estes são informados de que a TPS é tão importante quanto o tratamento periodontal ativo. Pesquisa recente realizada na Índia também mostrou esta alta adesão dos pacientes na TPS (SUBRAMANIUM et al., 2014). Todavia, outro estudo demonstra taxas de adesão muito inferiores aos encontrados nesta pesquisa (RAMSEIER et al., 2014). Em adição, tem-se uma pesquisa realizada em Pittsburgh, nos Estados Unidos, com 315 pacientes relatando que apenas 30\% deles retornaram para TPS. Vários fatores podem interferir na regularidade das visitas durante a TPS tais como a falta de conscientização do paciente sobre a importância destes retornos para que sua saúde periodontal seja mantida, eficácia e cooperação com a higiene bucal, número de dentes, condição sistêmica, frequência prévia à TPS, acesso à instrumentação, história de doença periodontal e profundidade de sondagem aumentada (FAMILI; SHORT, 2010).

Dentro desse contexto de cooperação à TPS, pode ser enfatizado que a aplicação de indicadores sócio-dentários para avaliar o impacto de determinada condição na qualidade de vida são utilizados para pesquisas sobre saúde bucal. Através do índice subjetivo OHIP-14 (Oral Health Impact Profile), foi observado que tanto no T1 quanto no T2, os pacientes colaboradores tiveram um impacto fraco em relação à doença periodontal. Levando em consideração este exposto, alguns trabalhos relataram que pacientes insatisfeitos com sua saúde bucal apresentaram maiores pontuações deste índice (PAPAGIANNOPOULOU et al., 2012; PIRES, 2009; OLIVEIRA; NADANOVSKY, 2005; LOCKER; ALLEN, 2002; LOCKER et al., 2001). Em relação aos resultados desta pesquisa, não houve escores de impacto forte, demonstrando uma boa percepção sobre a saúde bucal dos indivíduos.

A Terapia Periodontal de Suporte continua sendo uma forma de tratamento eficaz para o controle da doença periodontal após tratamento ativo, pois os pacientes que retornam para as consultas da TPS apresentam quadros clínicos e periodontais mais satisfatórios do que aqueles do diagnóstico inicial. Porém, a eficácia desta terapia depende tanto da colaboração do profissional quanto do paciente. Esta pesquisa teve como limitações a ausência de pacientes marcados às consultas, bem como a quantidade de números de telefones incorretos ou que tiveram seus números trocados, impossibilitando a comunicação necessária. Assim, novos desenhos de estudos são necessários, em especial, pesquisas longitudinais que possam avaliar mudança comportamental dos pacientes, os riscos individuais que interferem na resposta à TPS e 
os intervalos variados de tempo mais apropriados para o restabelecimento da saúde dos tecidos periodontais; logo conferindo novos achados que possibilitem contribuir ainda mais, por meio de terapias aplicadas efetivas, com a saúde periodontal de toda a população.

\section{CONCLUSÃO}

Os pacientes, em sua maioria, mostraramse colaboradores na Terapia Periodontal de Suporte, mantendo sua condição bucal estável ou progredindo para saúde periodontal. Os pacientes colaboradores apresentam melhores condições bucais quando comparados aos pacientes colaboradores irregulares ou não colaboradores.

\section{REFERÊNCIAS}

AMERICAN ACADEMY OF PERIODONTOLOGY. POsition paper: Periodontal maintenance. J Periodontol., v. 74, n. 9, p. 1395-1401, 2003.

ARMITAGE, G. C. Development of system for periodontal conditions. Ann Periodontol., v. 4, n. 1, p. 1-6, 1999.

BARDERSTEN, A.; NILvÉUS, R.; EGELBERG, J. Scores of plaque, bleeding, suppuration and probing depth to predict probing attachment loss. J Clin Periodontol., v. 17, n. 2, p. 102-107, 1990.

BASTOS, R. S. Impacto das condições de saúde bucal em relação à qualidade de vida de adolescentes escolares de 15 a 19 anos, numa dicotomia econômica, no município de Bauru. 2009. (Tese de Doutorado) - Universidade de São Paulo, Faculdade de Odontologia de Bauru, Bauru, São Paulo, 2009.

BELLO, D. M. A.; ARAÚJO, N. C.; GUSMÃO, E. S.; SOUZA, P. R. E.; DONOS, N.; CIMÕES, R. Condição Periodontal e Marcadores do Controle Metabólico em Pacientes Diabéticos. Pesqui Bras Odontoped Clín Integr., v. 11, n. 3, p. 357-361, 2011.

BUISCHI, Y. P. Promoção de saúde bucal na clínica odontológica. São Paulo: Artes Médicas: EAP-APCD, 2000.
CLAFFEY, N.; NYLUND, K.; KIGER, R.; GARRET, S.; EGELBERG, J. Diagnostic oredictability of scores of plaque, bleeding, suppuration and probing pocket depths for probing attachment loss. 3 years os observation folloeing initial therapy. J Clin Periodontol., v. 17, n. 2, p. 108-114, 1990.

CALVASINA, P. G.; NUTO, S. A. S. A aliança na terapia periodontal de suporte: avaliando a motivação e o autocuidado na periodontite crônica. R. Periodontia, v. 19, n. 2, p. 54-63, 2009.

CARRANZA JUNIOR, F. A.; NEWMAN, M. G.; TAKEY, H. H.; KLOKKEVOLD, P. R. Periodontia clínica. Rio de Janeiro: Elsevier, 2011.

COSTA, F. O.; LAGES, E. J.; COSTA, L. O.; LORENTZ, T. C.; SOARES, R. V.; CORTELLI, J. R. Tooth loss in individuals under periodontal maintenance therapy: 5 year prospective study. J Periodontal Res., v. 49, n. 1, p. 121-8, 2014.

DANTAS, T. S.; LELIS, É. R.; FERREIRA, J. M.; RENOVATO, S. R.; NAVES, L. Z.; FERNANDES-NETO, A. J. Terapia periodontal de suporte: objetivos, procedimentos e intervalos. Rev. UNOPAR Cient. Ciênc. Biol. Saúde., v. 13, n. 3/4, p. 369-74, 2011.

DEMIREL, K; EFEODLU, A. Retrospective evaluation of patient compliance with supportive periodontal treatment. J Nihon Univ Sch Dent., v. 37, n. 3, p. 131137, 1995.

DRUMOND-SANTANA, T.; COSTA, O. F.; ZENÓBIO, E. G.; SOARES, R. V.; SANTANA, T. D. Impacto da doença periodontal na qualidade de vida de indivíduos diabéticos dentados. Cad. Saú. Púb., Rio de Janeiro. v. 23, n. 3, p. 637-644, 2007.

FAMILI, P.; SHORT, E. Compliance with periodontal maintenance at the University of Pittsburgh: retrospective analysis of 315 cases. Gen Dent., v. 58, n. 1, p. 42-7, 2010.

GRANDE, F. Z.; ALVES, F. B. T.; GRANDE, C. Z.; SANTOS, F. A.; PILATTI, G. L. Terapia periodontal de suporte objetivos, procedimentos e intervalos. Rev. UEPG Cien. Biol., v. 13, n. 3/4, p. 15-22, 2007. 
GUGLIELMETTI, M. R.; GANHITO, J. Á.; CARVALHO, C. V.; ROMITO, G. A.; CONDE, M. C.; MICHELI, G. Cooperação consciente: Programa de Motivação aos Retornos Periódicos Periodontais. Rev Periodontia, v. 20, n. 2, p. 07-12, 2010.

HABER, J.; KENT, R. L. Cigarette smoking in periodontal practice. J Periodontol., v. 63, n. 2, p. 100-106, 1992.

JONSSON, B.; BAKER, S. R.; LINDBERG, P.; OSCARSON, $\mathrm{N}$.; OHRN, K. Factors influencing oral hygiene behaviour and gingival outcomes 3 and 12 months after initial periodontal treatment: an exploratory test of an extended Theory of Reasoned Action. J Clin Periodontol., v. 39, n. 2, p. 138-144, 2012.

JOSS, A.; ADLER, R.; LANG, N. P. Bleeding on probing. A parameter for monitoring periodontal conditions in clinical practice. J Clin Periodontol., v. 21, n. 6, p. 402408, 1994.

LAURELL, L.; LUNDGREN, D.; FALK, H.; HUGOSON, A. Long-term prognosis of extensive polyunit cantilevered fixed partial dentures. J Prosthet Dent., v. 66, n. 4, p. 545-52, 1991.

LINDHE, J.; LANG, N. P.; KARRING, T. Tratamento de periodontia clínica e implantodontia oral. 5. ed. Rio de Janeiro: Guanabara Koogan, 2010.

LINDHE, J.; NYMAN, S. Long-term maintenance of patientes treated for advanced periodontal disease. J Clin Periodontol., v. 11, n. 8, p. 504-14, 1984.

LOCKER, D.; ALLEN, P. F. Developing short-form measures of oral health-related quality of life. J Pub Health Dent., v. 62, n. 1, p. 13-20, 2002.

LOCKER, D.; MATEAR, D.; STEPHENS, M.; LAWRENCE, H.; PAYNE, B. Comparison of the GOHAI and OHIP-14 as measures of the oral health-related quality of life of the elderly. Community Dent Oral Epidemiol., v. 29, n. 5, p. 373-81, 2001.

LORENTZ, T. C. M.; COSTA, L. O. M.; CORTELLI, J. R.; VARGAS, A. M. D.; COSTA, F. O. Prospective study of complier individuals under periodontal maintenance therapy: analysis of clinical periodontal parameters, risk predictors and the progression of periodontitis. $\mathbf{J}$ Clin Periodontol., v. 36, p. 58-67, 2009.

MARSH, P. D. Dental plaque as a microbial biofilm. Caries Res., v. 38, n. 3, p. 204-11, 2004.

MIOTTO, M. H. M. B.; BARCELLOS, L. A.; VELTEN, D. B. Avaliação do impacto na qualidade de vida causado por problemas bucais na população adulta e idosa em município da Região Sudeste. Ciênc Saú Coletiva, v. 17, n. 2, p. 397-406, 2012.

MLACHKOVA, A. M.; POPOVA, C. L. Efficiency of non surgical periodontal therapy in moderate chronic periodontites. Folia Med., v. 56, n. 2, p. 109-15, 2014.

O'LEARY, T. J.; DRAKE, R. B.; NAYLOR, J. E. The plaque control record. J Periodontol., v. 43, n. 1, p. 38-48, 1972.

OLIVEIRA, B. H.; NADANOVSKY, P. Psychometric properties of the Brazilian version of the Oral Health Impact Profile - Short form. Comm Dent Oral Epidemiol., v. 33, n. 4, p. 307-314, 2005.

PADOVANI, M. C.; SABA-CHIJFI, E. Manutenção e controle no tratamento periodontal. APCD., v. 45, n. 2, p. 429432, 1991.

PAPAGIANNOPOULOU, V.; OULIS, C. J.; PAPAIOANNOU, G. A.; ANTONOGEORGOS, G.; YFANTOPOULOS, J. Validation of a Greek version of the oral health impact profile (OHIP-14) for use among adults. Health Qual Life Outcomes., v. 10, n. 7, p. 1-10, 2012.

PEREIRA, A. F. V.; PAIVA, M. T. A.; COSTA, L. A.; BRASILIENSE, F. P. H. D.; SANTOS, F. F. C.; LEITE, S. A. M. Visão crítica da fase de manutenção no contexto da terapia periodontal. Odontol Clin-Cient., v. 10, n. 1, p. 25-29, 2011.

PIRES, I. C. G. R. A Influência da saúde oral na qualidade de vida. 2009. Dissertação (Doutorado) Faculdade de Medicina dentária da Universidade do Porto, Portugal, 2009.

RAMSEIER, C. A.; KOBREHEL, S.; STAUB, P.; SCULEAN, A.; LANG, N. P.; SALVI, G. E. Compliance of cigarette 
smokers with scheduled visits for supportive periodontal therapy. J Clin Periodontol., v. 41, p. 473-480, 2014.

RENVERT, S.; PERSSON, G. R. Supportive periodontal therapy. Periodontol 2000, v. 36, p. 179-95, 2004.

SAMINSKY, M.; HALPERIN-STERNFELD, M.; MACHTEI, E. E.; HORWITZ, J. Variables affecting tooth survival and changes in probing depth: a long-term follow-up of periodontitis patients. J Clin Periodontol., v. 42, p. 513$519,2015$.

SILVA, A. M.; VARGAS, A. M. D.; FERREIRA, E. F.; ABREU, M. H. N. G. A integralidade da atenção em diabéticos com doença periodontal. Cienc Saúde Coletivos, v. 15, n. 4, p. 2197-206, 2010.

SILVA, S. R. C.; FERNANDES, R. A. C. Autopercepção das condições de saúde bucal por idosos. Rev Saúde Pública., v. 35, n. 4, p. 349-355, 2001.

SLADE, G. D. Derivation and validation of a short-form oral health impact profile. Comm Dent Oral Epidemiol., v. 25, n. 4, p. 284-290, 1997.

SOCRANSKY, S. S.; HAFFAJEE, A. D. Periodontal microbial ecology. Periodontol 2000, v. 38, p. 135-187, 2005.

SOUSA, J. N. L.; NÓBREGA, D. R. M.; ARAKI, A. T. Perfil e percepção de diabéticos sobre a relação entre diabetes e doença periodontal. Rev Odontol UNESP., v. 43, n. 4, p. 265-272, 2014.

SUBRAMANIUM, G.; NATARAJAN, B.; RAMARAJ, J. A.; KAVIN, T. Patient compliance and supportive periodontal therapy: Study among young adults of Namakkal district. J Pharm Bioallied Sci., v. 6, n. 1, p. 171-173, 2014.

VALDERHAUG, J. Periodontal conditions and carious lesions following the insertion of fixed prostheses: a 10year follow-up study. Int Dent J., v. 30, n. 4, p. 296-304, 1980.

WEHMEYER, M. M. H.; CORWIN, C. L.; GUTHMILLER, J. M.; LEE, J. Y. The impact of oral health literacy on periodontal health status. J Public Health Dent., v. 74, p. 80-87, 2014.
WESTFELT, E.; NYMAN, S.; SOCRANSKY, S.; LINDHE, J. Significance of frequency of professional tooth cleaning for healing following periodontal surgery. J Clin Periodontol., v. 10, n. 2, p. 148-56, 1983.

Recebido em: 05 de setembro de 2015 Aceito em: 13 de outubro de 2015 